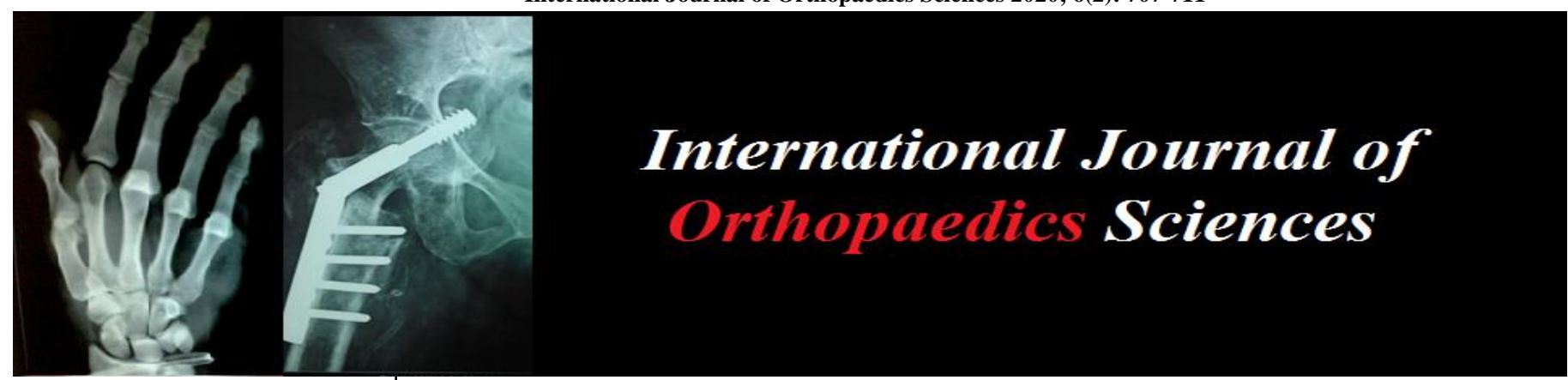

E-ISSN: 2395-1958

P-ISSN: 2706-6630

IJOS 2020; 6(2): 707-711

(C) 2020 IJOS

www.orthopaper.com

Received: 01-02-2020

Accepted: 03-03-2020

Dr. Mohammed Shoaib Qureshi Department of Orthopedics, MGM Hospital \& Research Centre, Aurangabad,

Maharashtra, India

Dr. Mangesh Panat

Department of Orthopedics, MGM Hospital \& Research

Centre, Aurangabad,

Maharashtra, India

\section{An assessment of distraction plating in the management of intra-articular comminuted distal end radius fractures in the elderly patients}

\author{
Dr. Mohammed Shoaib Qureshi and Dr. Mangesh Panat
}

DOI: https://doi.org/10.22271/ortho.2020.v6.i21.2124
Corresponding Author: Dr. Mohammed Shoaib Qureshi Department of Orthopedics, MGM Hospital \& Research Centre, Aurangabad, Maharashtra, India

\begin{abstract}
Background: Distal radius fractures are one of the most common injuries representing one sixth of all fractures seen and treated in emergency department. Distal radial fractures with extensive comminution involving the metaphyseal-diaphyseal junction present a major treatment dilemma specially when it has an intra-articular involvement. This study evaluates the surgical and functional outcomes of IntraArticular comminuted fracture of Distal End Radius treated with dorsal distraction plating.

Materials and Methods: A prospective randomized controlled observational study was done with a sample size of 50 patients over a period of 3 years each of them diagnosed to be with Intra-articular Fracture of Distal End Radius (AO Classification Type C).All patients were treated by distraction plating (Radio-Metacarpal). Minimum follow up after treatment up to 6 months was ensured and final assessment of functional outcome was then decided.

Results: The study showed that average union time was $8.65 \pm 0.93$ weeks and average implant removal was done at $8.75 \pm 0.85$ weeks. All patients achieved full ROM at wrist maintaining articular congruity post implant removal. In our study we found two $(6.67 \%)$ patients with superficial infection; and two (6.67\%) patients with Complex Regional Pain Syndrome (CRPS). No patients were reported with tendon (Extensor pollicis longus) irritation, neuropraxia, stiffnes of wrist and interphalangeal joints, nonunion/malunion and implant failure.

Conclusion: The final results were evaluated and compared after making observations for age, sex, mode of trauma, dominancy of affected hand, time interval between injury and admission, duration of hospital stay, duration of surgery, time to fracture union and complications. The final outcome was measured by DASH SCORE. All patients had excellent or good results treated with distraction plating; six (20.00\%) patients out of 30 studies found to have excellent result whereas remaining Twenty four $(80.00 \%)$ patients had good result.
\end{abstract}

Keywords: Intra-articular communited distal end radius fracture, distraction plate

\section{Introduction}

Distal radius fractures are one of the most common injuries encountered in orthopedic practice [1]. They make up $8 \%-15 \%$ of all bony injuries in adults. The treatment goals for the management of these fractures include restoring congruity to the radiocarpal and distal radioulnar joint (DRUJ) surfaces and maintaining radial length. Currently, there are a number of established surgical options for displaced distal radius fractures as well as newer novel approaches being developed for the surgical management of these injuries ${ }^{[2,3]}$. Distal radial fractures with extensive comminution involving the metaphyseal-diaphyseal junction present a major treatment dilemma specially those involving the articular surface. The use of internal distraction plating or bridge plating for distal radius fractures was first introduced by Burke and Singer ${ }^{4}$. The technique was further expanded by Ruch and coworkers, who described the use of a 12- to 16-hole 3.5-mm dynamic compression plate (DCP) (Synthes, Paoli, PA) placed in the floor of the fourth dorsal extensor compartment to span from the intact radius diaphysis to the third metacarpal ${ }^{[5,6]}$. The bridge plating technique provides strong fixation and allows for distraction across impacted articular segments. The technique can be combined with a limited articular fixation approach for those fracture patterns with intra-articular extension. Ever since Abraham colles 7 published "on the fracture of the carpal extremity of the radius" 
in Edinburgh medical journal (1814), no other fracture has been discussed about as much ${ }^{[7]}$. This study evaluates the surgical and functional outcomes of Intra-Articular communited Fracture of Distal End Radius treated with dorsal distraction plating.

\section{Materials and Methods}

This was a prospective randomized controlled observational study with a sample size of 50 patients. Population included all patients with Intra-articular Fracture of Distal End Radius (AO Classification Type C) presenting to the Orthopedic Department of MGM Medical College \& Hospital, Aurangabad (Maharashtra) during the study period from November 2015 to October 2017. Patients were treated by Close reduction with dorsal distraction plating. All surgical procedures were done in the operation theatre under strict aseptic precautions and under suitable anesthesia. Minimum follow up after treatment up to 6 months was ensured for all patients included in the study. They were followed up and assessed at every 2, 6, 8, 10 and 12 weeks for fracture union, status of implant, finger and wrist movements and complications. Final assessment of functional outcome was done at 6 months. Final results were assessed using the DASH Score. Implant (plate) removal shall be done at 12 weeks or after radiological fracture union and wrist mobilization was started.

\section{Inclusion \& Exclusion Criteria \\ Inclusions}

- Intra articular comminuted DER fractures (AO type $23 \mathrm{C}$ 1,2 and 3) where fracture fragments are too small to be fixed by distal-radius plate.

- Close fractures.

- Age more than 40 years.

- Good skin condition.

\section{Exclusions}

- Patients having Extra-articular fracture Distal end Radius \& Segmental Radius fracture.

- Open fractures.

- Age less than 40 years.

- Patients having fracture of any carpal bones.

- Patients with Neurovascular compromise/injury.

\section{Surgical Technique}

Position: The patient is positioned supine by placing the forearm on a side arm support. A non-sterile pneumatic tourniquet is used. Prophylactic antibiotics are given intravenously $30 \mathrm{mins}$ prior to skin incision.

Technique: The surgical approach involves 2 or 3 incisions. The first is performed over the midshaft of the third metacarpal. A second incision measuring approximately $4 \mathrm{~cm}$ is required at the dorsal aspect of the radius at least $4 \mathrm{~cm}$ from the most proximal portion of the comminuted fracture. The extensor mechanism is retracted after the first incision. A 2.7$\mathrm{mm}$ or $3.5-\mathrm{mm} \mathrm{DCP/LCP}$ is then passed along the surface of the bone from the distal to the proximal incision. A 12-14-16hole plate can be selected. However, plate selection should permit the insertion of a minimum of 3 cortical screws proximally. The Third incision (if required), approximately 2 $\mathrm{cm}$ in length, is made over the Lister's tubercle to facilitate mobilization of the extensor pollicis longus (EPL) tendon. This helps the passage of the plate under the extensor tendons. Complete exposure \& release of the EPL is performed to verify that the plate lies underneath the tendons, and to facilitate placement of grafts, because this incision can also be used as a portal for placement of allograft bone in the metaphyseal area if a defect is present. Supplemental bone grafting is delayed in cases of open injuries to avoid the risk of infection. The EPL is then exposed, and the position of the plate is re-evaluated to ensure that the plate does not impinge on either the EPL or the digital extensors. Afterward, the plate is fixed to the long finger metacarpal. The hole must be drilled at the midline of the metacarpal to avoid any rotatory displacement. Traction is then applied manually under fluoroscopic visualization to obtain radial length. The plate is then fixed proximally in neutral rotation. The wrist must be in neutral position. The diaphyseal fragments are then reduced and fixed to the shaft. Interfragmentary screws can be valuable where necessary. The restoration of the articular surface of the radius is achieved percutaneously. In case of difficulties, a small dorsal incision may be helpful to ensure adequate length of the radial column. Anatomic reduction may require the placement of a cancellous screw through the plate into the reduced articular segments. Sometimes, the distal fragments are too small for screws. In this case, Kirschner wires can be used under fluoroscopy to reduce the articular surface. In case pins were used, they have to be removed at 6 weeks. Implant (plate) removal shall be done at 8-10 weeks or after radiological fracture union and wrist mobilization was started.

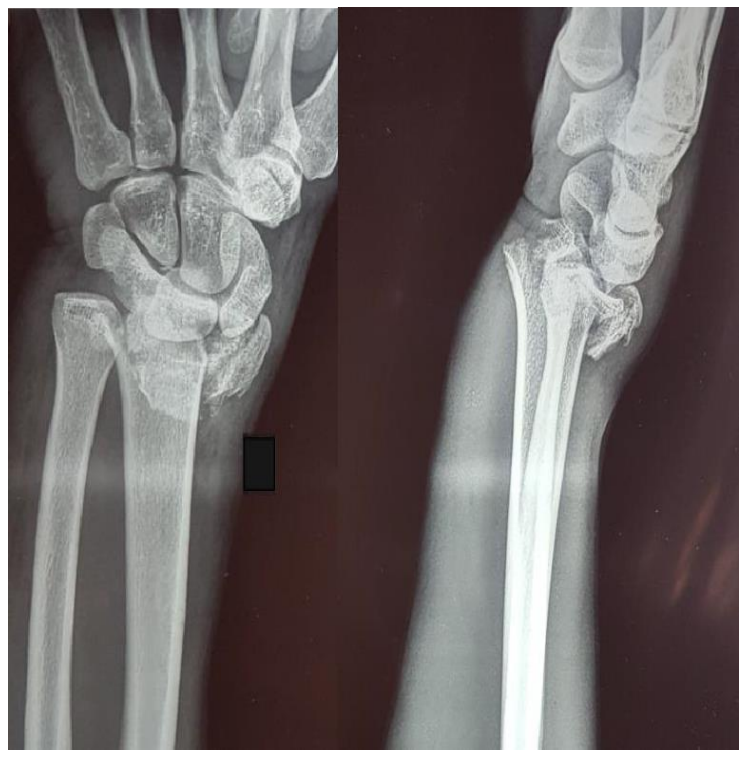

Pre OP -RAY

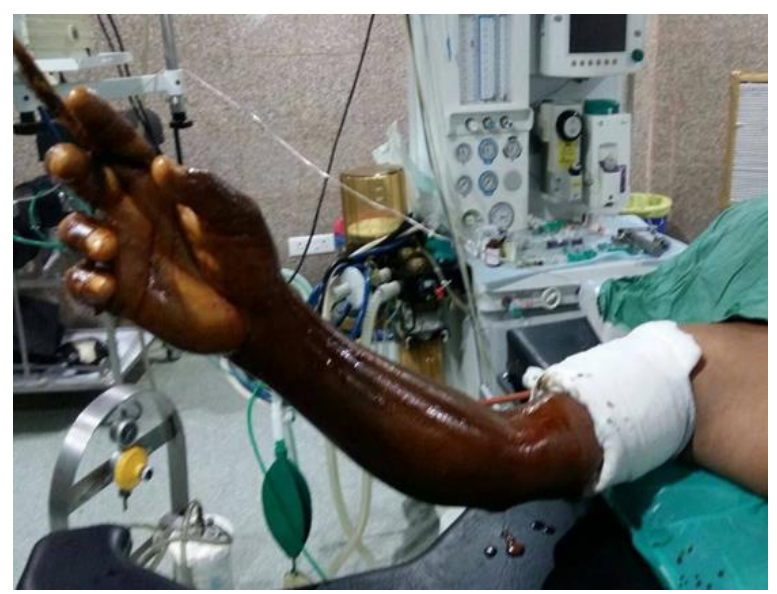

Non-Sterile Pneumatic Tourniquet Applied 


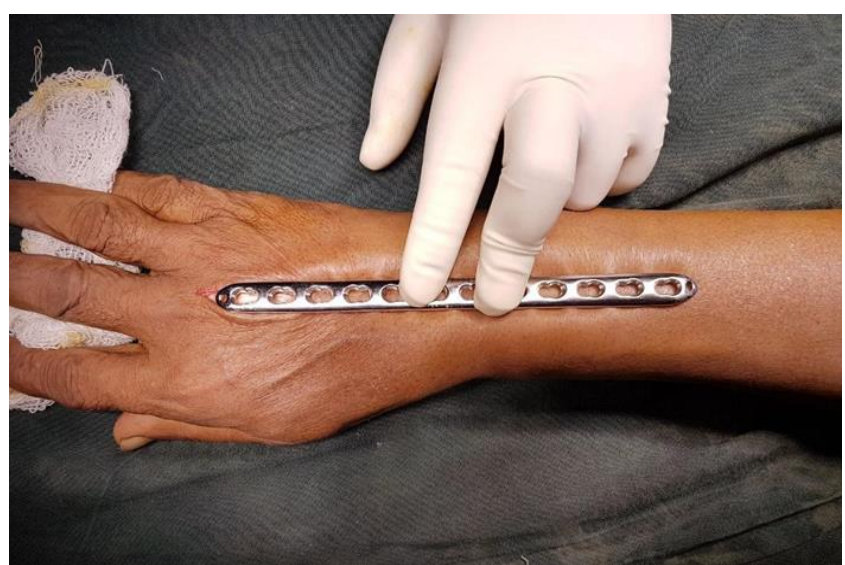

Measuring of The Plate

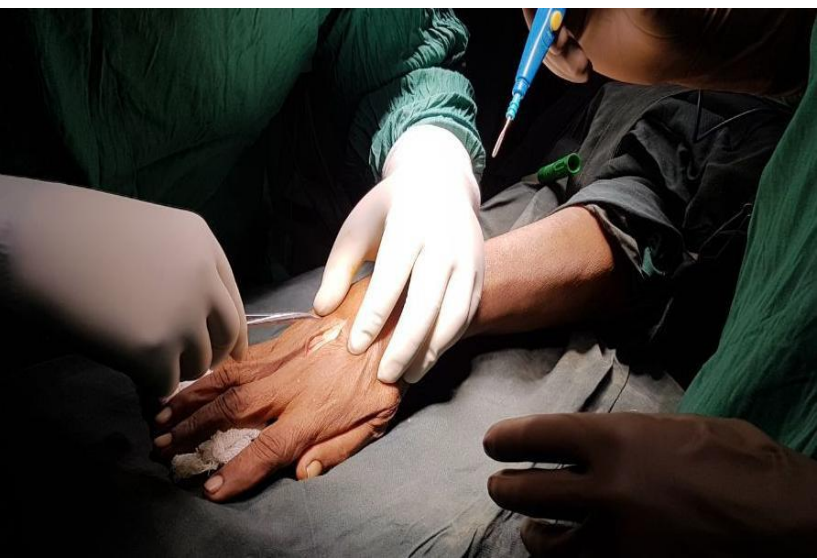

First Incision Over 3rd Metacarpal

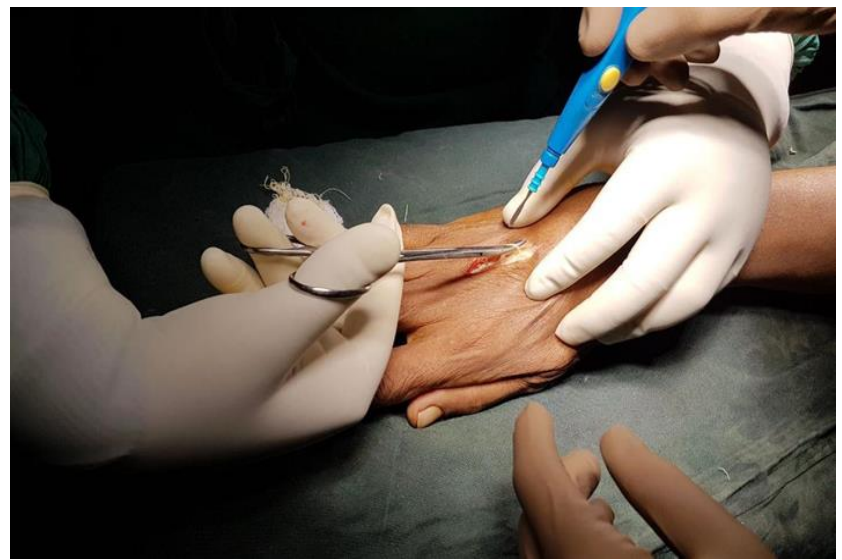

Dissection Upto The Metacarpal



Creating A Tunnel Through Cobbs Elevator

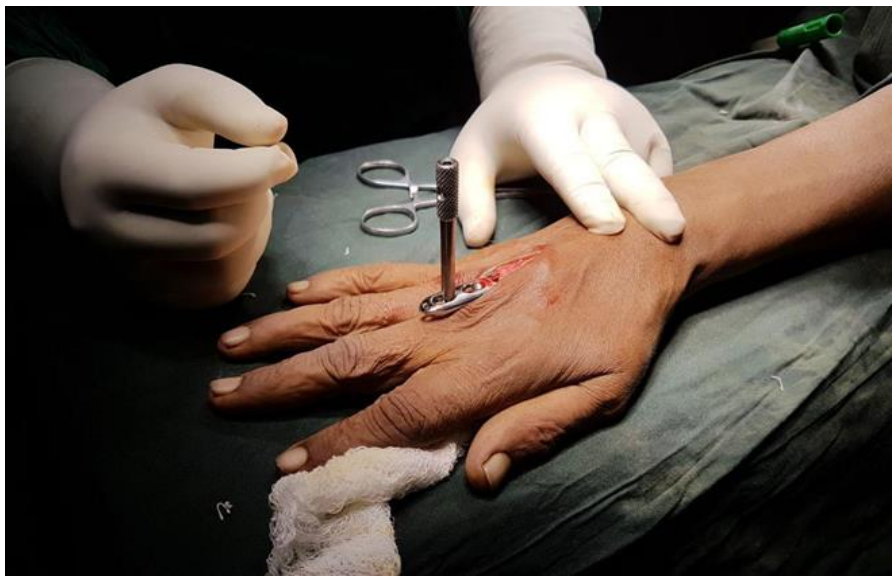

Placing Plate Through First Incision



Placing The Plate Underneath The Extensor Pollicis Longus Belly

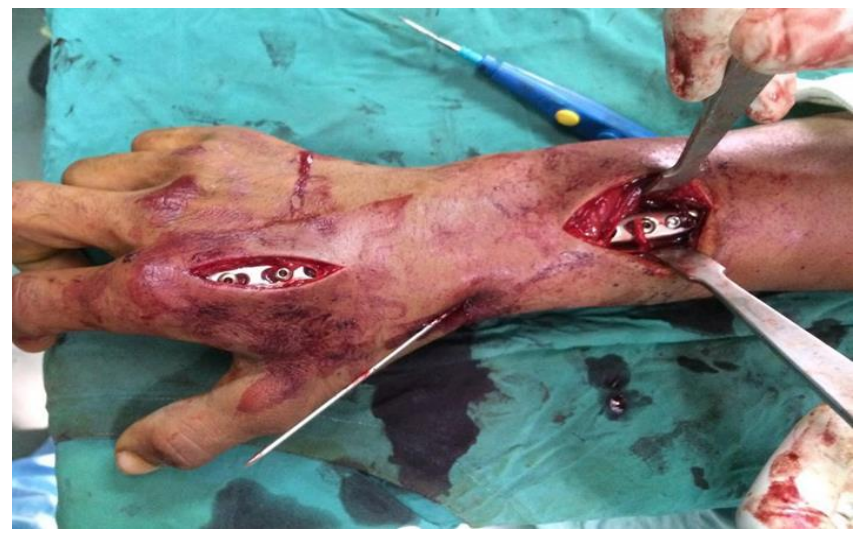

Adequate Sized Screws Fixed With Temporary K-Wire Fixation (If Required)

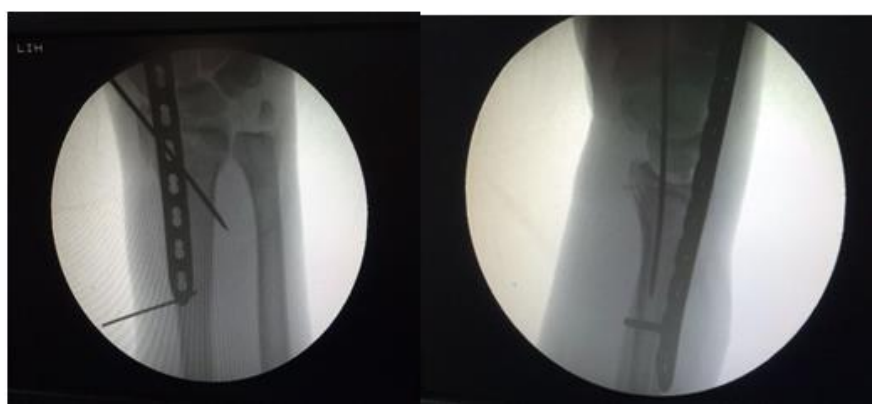

Intraoperative Fluoroscopy Images 


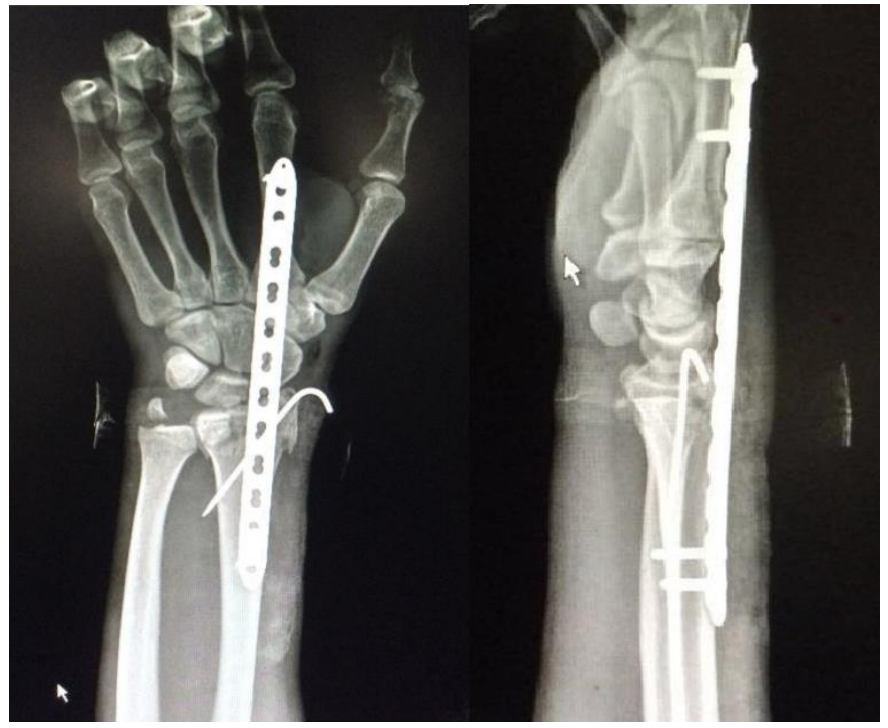

Post-Op Xray AP \& Lateral View



Post Implant Removal X-Ray Left Wrist AP \& Lat View (9 Weeks)

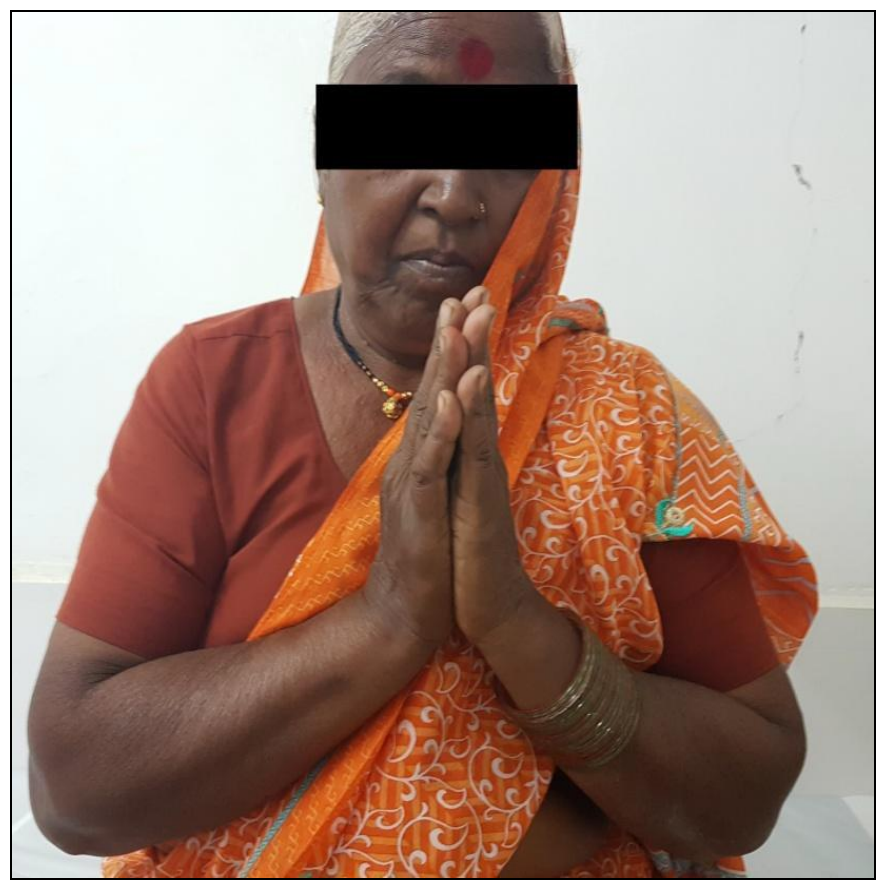

Range Of Motion - Extension (12 Weeks Post-Op)

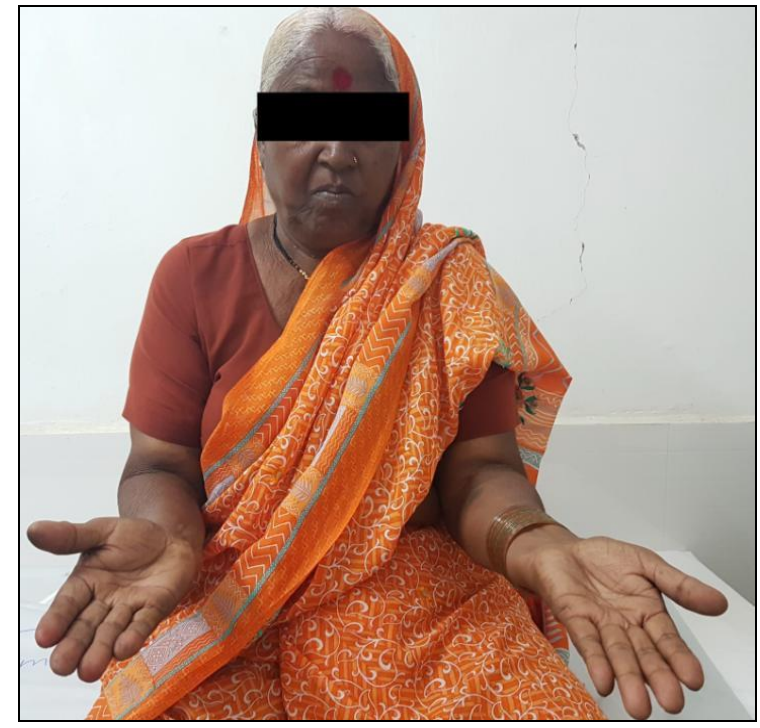

Range of Motion - Supination (12 Weeks Post-Op)

\section{Results}

Of the 30 patients, average age was 55.26 \pm 5.96 years. According to AO classification, there were 07(23-C1), 16(23$\mathrm{C} 2$ ) and 07(23-C3) type of fracture. The patients were predominantly males $16(53.33 \%)$. The dominant hand was injured in $16(53.33 \%)$. Patients presented with various mode of trauma like 06 were fall at home, 03 were fall at farm, 08 had fall from bike, 01 had fall from height, 09 had fall from stairs and 03 were hit by bike. Three patients had associated injuries such as proximal tibia fracture, intertrochanteric femur fracture \& bimalleolar fracture. Average injury to surgery duration was $2.91 \pm 1.15$ days with average operative time of $48.09 \pm 10.37$ minutes. In our study we found $2(6.67 \%)$ patients with superficial infection; and 2(6.67\%) patients with Complex Regional Pain Syndrome (CRPS). No patients were reported with tendon (Extensor pollicis longus) irritation, neuropraxia, stiffness of wrist and inter- phalangeal joints, non-union and malunion, and implant failure. Post implant removal wrist manipulation was done under anesthesia and post-operatively wrist ROM was started which subsequently results in full ROM at wrist along with maintaining articular congruity. Average union time was $8.65 \pm 0.93$ weeks. And average implant removal was done at $8.75 \pm 0.85$ Weeks. In our study all of patients had excellent or good results treated with distraction plating; $6(20.00 \%)$ patients out of 30 studies found to have excellent result whereas remaining 24(80.00\%) patients had good result. In our study we found Mean \pm SD DASH SCORE of $23.25 \pm 2.09$.

\section{Discussion}

The stabilization of highly comminuted metaphysealdiaphyseal fractures of the distal part of the radius remains a major orthopedic challenge as a result of both biological and biomechanical considerations. High-energy fractures with extensive comminution often require fixation for a prolonged period of time in order to achieve union. In Intra articular comminuted DER fractures ( AO type $23 \mathrm{C} 1,2$ and 3) where fracture fragments are too small to be fixed by distal-radius plate and also prolonged immobilization with external fixation is often not possible because of pin-track infection or loosening leading to loss of reduction, stiffness and over distraction, the use of the distraction plate permitted the softtissue envelope to mature while maintaining radial length, allowing retaining congruity of the radiocarpal and radioulnar joints. 
We believe that the dorsal ulnar position of this plate gives it an advantage over conventional external fixation, which relies on maintaining the length of the ulnar column of the radius (metaphyseal bone underlying the lunate fossa) from a radially based axis. With plate fixation on the long-finger metacarpal, the distraction moment is centralized with the added advantage of stabilizing the lunate fossa of the distal part of the radius. In addition, the implant serves simultaneously as a distraction device and a dorsal buttress to the fracture. Radial length is maximized and radiocarpal alignment and palmar tilt are maintained. Carpal alignment and the alignment of the carpus with respect to the distal part of the radius have been demonstrated to be an important determinant of functional outcome after distal radial fracture. This alignment was accomplished in our patients and may account for the satisfactory range of motion and functional results. We believe that another advantage of the dorsal distraction-plate fixation technique is that it permits the patients to use the upper extremity for moderate or light activities while the comminuted segments heal. An external plaster splint is not required.

Three of the thirty patients had associated injuries. The stability of the implant permitted those patients to use a platform walker to assist in transfers during physical activity and to perform personal hygiene. Furthermore, thirteen of thirty patients who had been working before the injury were able to return to light duty work during their treatment.

There are two distinct concerns with the use of this technique. First, there is the potential for stiffness of the wrist. The patients in this series all had substantial comminution, which would have necessitated some protective immobilization regardless of the fixation technique that was employed. The average time that the plate remained in place was $8.53 \pm 0.85$ weeks in our study.

Our study suggests that a functional range of motion can be regained within the three months after the injury despite up to two months of plate fixation across the wrist joint. Taken together, these findings suggest that the implant can remain in place across the wrist joint until union occurs without risking any more loss of motion than can be expected on the basis of the severe nature of the injury. The other concern regarding this technique is the potential for delayed union of the radius

\section{Conclusion}

Finally, we would like to conclude that internal distraction pating is a simple, easy, reliable and more suitable method of treatment in some distal end radial fractures. It is a lesser invasive percutaneous method, hence chances of iatrogenic muscle /tendon impairement and neurovascular injury tends to diminish too. Dorsal distraction plate fixation of dorsally unstable distal radius communited small fractures is a new method of treatment that provides the benefits of stable internal fixation. We recommend this device as an alternative method to the currently used modalities and not as a superior substitute keeping the limitations of this technique in mind. It is essentially the concept of moving external fixation into the subcutaneous space to avoid the problems with pin tract infections \& to increase patient acceptability. The current technique provides excellent screw purchase in osteopenic bone because the device is secured to the cortical bone of the long metacarpal \& the radius.

Early post-operative mobilization is possible in the patients treated with CRIF and plating. Distraction plating appeared to provide acceptable results based on the ability to restore functional range of motion and achieve acceptable radiographic outcomes.

A functional range of motion with minimal disability can be achieved despite a prolonged period of fixation with a distraction plate across the wrist joint, the range of motion and DASH scores were acceptable at the time of final followup.

This study adds to the growing body of evidence showing the significant fracture stability that can be achieved with a radiocarpal-spanning plate. It further shows that applying the plate with the wrist in slight extension does not significantly compromise this stability. It is our opinion that having an immobilized wrist in a more functional position could greatly benefit patients.

The only relative disadvantage of the procedure is the requirement of an additional surgery for the removal of the plate after fracture union. The use of a more rigid method of maintaining the reduction may prevent the collapse that would otherwise permit union.No hardware failures occurred in this series, and there were no cases of extensor tendon injury or tenosynovitis as have been previously reported with use of a conventional dorsal plate.

Declaration of patient consent: We certify that the patients included in this study had no objections whatsoever have their images and clinical information being included in this study. The identity of these patients have also been concealed with all due efforts but complete anonymity cannot be guaranteed.

\section{Reference}

1. Graff S, Jupiter J. Fracture of distal radius: classification of treatment and indication for external fixation. Injury. 1994; 25:S-D14-S-D25.

2. Drobetz H, Bryant AL, Pkorny T et al: volar fixed-angle plating of distal radius extension fractures: influence of plate position on secondary loss of reduction-a biomechanic study in a cadaveric model. J hand Surg [Am]. 2006; 31:615-622.

3. Tornetta P3 ${ }^{\text {rd }}$, Klein DM, Stein AB, McQueen M. Distal radius fracture. J Orthop Trauma. 2002; 16:608-611.

4. Burke EF, Singer RM. Treatment of communites distal radius with the use of an internal distraction plate. Tech Hand Up Extrem Surg. 1998; 2:248-252.

5. Ginn TA, Ruch DS, Yang CC, Hanel DP. Use of a distraction plate for distal radial fractures with metaphyseal and diaphyseal communition: surgical technique. J Bone Joint Surg AM. 2006; 88(1):29-36.

6. Ruch DS, Ginn TA, Yang CC et al:: Use of a distraction plate for distal radial fractures with metaphyseal and diaphyseal comminution. J Bone Joint Surg AM. 2005; 87:945-954.

7. Colles A. On the fracture of the carpal extremity of the radius. Edinburgh Med Surg. 1814; 10:182-186. 